Bio - grafia. Escritos sobre la Biología y su Enseñanza. ISSN 2027

Edición Extraordinaria. p.p. $871-881$

Memorias del VIII Encuentro Nacional de Experiencias en Enseñanza de la Biología y la Educación Ambiental. III Congreso Nacional de Investigación en Enseñanza de la Biología.

\title{
FORMACIÓN EN INVESTIGACIÓN EN FUTUROS PROFESORES. EL CASO DE SEXTO SEMESTRE DE LICENCIATURA EN BIOLOGIAA DE LA UNIVERSIDAD PEDAGÓGICA NACIONAL. PLANTEAMIENTO DEL PROBLEMA ${ }^{1}$
}

\section{FUTURE RESEARCH TRAINING TEACHERS. THE CASE OF SIXTH SEMESTER DEGREE IN BIOLOGY NATIONAL UNIVERSITY TEACHING. PROBLEM}

0

$\mathrm{N}$

E

N

C

I

A

\section{RESUMEN}

Natalia Valencia ${ }^{2}$

Edgar Valbuena ${ }^{3}$

A continuación se presenta una propuesta investigativa que se encuentra en el marco del proyecto de investigación denominado "Formación en investigación en futuros profesores de Biología" de la línea de investigación Conocimiento Profesional del Profesor de Ciencias; este proyecto tiene como objetivo caracterizar la formación investigativa en futuros profesores de biología (sexto semestre) de la Licenciatura en biología de la Universidad Pedagógica Nacional. Específicamente, se describe el problema, los fundamentos teóricos centrados en la formación investigativa de profesores y la metodología propuesta.

\section{ABSTRACT}

Here we present a research proposal which is under the research project entitled" Research Training future teachers of Biology" of the research of Professor Professional Knowledge Science, this project aims to characterize the formation future research in biology teachers (sixth semester) of the degree in biology from the National Pedagogical University. Specifically, it describes the problem, theoretical research focused on teacher training and the proposed methodology.

\section{PALABRAS CLAVE}

Formación en investigación en profesores de ciencias, PCLB.

\footnotetext{
${ }^{1}$ Propuesta construida para optar a la convocatoria de jóvenes investigadores de Colciencias, en el marco del proyecto de investigación denominado "Formación en investigación en futuros profesores de Biología"

2 Licenciada en Biología. Universidad Pedagógica Nacional. Línea de investigación conocimiento profesional del profesor natacmona@hotmail.com

${ }^{3}$ Universidad Pedagógica Nacional, Colombia. Doctor en Didáctica de las Ciencias Experimentales. Director del Departamento de Biología. Coordinador del Grupo de Investigación Conocimiento Profesional del Profesor de Ciencias. valbuena@pedagogica.edu.co
} 
Bio - grafia. Escritos sobre la Biología y su Enseñanza. ISSN 2027

Edición Extraordinaria. p.p. $871-881$

Memorias del VIII Encuentro Nacional de Experiencias en Enseñanza de la Biología y la Educación Ambiental. III Congreso Nacional de Investigación en Enseñanza de la Biología.

\section{KEYWORDS}

Research Training in science teachers, PCLB

\section{INTRODUCCIÓN}

La presente propuesta se encuentra en el marco de la línea de investigación Conocimiento Profesional del Profesor de Ciencias (CPPC) de la Universidad Pedagógica Nacional (UPN), la cual se encarga de la producción de conocimiento y reflexión en torno al conocimiento profesional de los profesores, buscando la dignificación académica cultura y social de la profesión docente.

La investigación juega un papel muy importante en la formación de futuros profesores ${ }^{4}$ de Biología, puesto que les permiten, sistematizar, reflexionar e investigar su práctica pedagógica, dicho ejercicio promueve la construcción de conocimiento en torno a la didáctica de las ciencias, las prácticas educativas y el fortalecimiento de la identidad de la profesión docente. Por otro lado, la formación investigativa es importante para la profesión docente, dado que promueve actitudes que benefician la identidad profesional y permiten disminuir la subestimación de la profesión docente, además de esto la formación inicial de los profesores presentan gran influencia en las prácticas profesionales de los profesores, dado que desde alli se construye la identidad, las actitudes y aptitudes que orientan el ejercicio de la profesión docente. Estos aspectos son igualmente influyentes y determinantes en la educación y por ende benefician o afectan el desarrollo del país.

Esta propuesta busca la caracterización de la formación investigativa en futuros profesores de Biología (sexto semestre) de la Licenciatura en biología de la Universidad Pedagógica Nacional, con el fin de fortalecer la profesión docente y empoderamiento por parte de los futuros docentes de la construcción del conocimiento profesional como profesores de las ciencias, la reflexión de la práctica y la reorientación de las prácticas educativas para el mejoramiento de la escuela. Para lo anterior es describir la construcción del proyecto de integración (desde la perspectiva de formación en investigación) que realizan los estudiantes de sexto semestre con el fin de identificar las principales tendencias de formación en investigación. Además necesario indagar las concepciones de

\footnotetext{
4 En el desarrollo de la propuesta las expresiones; profesores en formación, estudiantes de la licenciatura en biología y profesores en formación inicial se entienden como sinónimos. La expresión formadores corresponde a los profesores de los estudiantes de la licenciatura e Biología de la Universidad Pedagógica Nacional
} 
Bio - grafia. Escritos sobre la Biología y su Enseñanza. ISSN 2027

Edición Extraordinaria. p.p. $871-881$

Memorias del VIII Encuentro Nacional de Experiencias en Enseñanza de la Biología y la Educación Ambiental. III Congreso Nacional de Investigación en Enseñanza de la Biología.

los formadores y los estudiantes de sexto semestre, sobre la investigación ${ }^{5}$ en el marco de la formación de profesores.

\section{MARCO TEÓRICO}

A continuación se presentarán algunos aspectos teóricos relevantes para la presente propuesta, como la formación docente, los modelos de formación docente, la formación en investigación de profesores y la formación investigativa en el PCLB.

\section{Formación docente}

Meinardi (2010), señala que la formación inicial de profesores, debe potenciar elementos del desarrollo profesional, realizando un proceso continuo, flexible y contextualizado, que se apoye en la reflexión de los aspectos sociales, éticos y políticos. Este proceso es posible a través de la producción de conocimiento a partir de la investigación en didáctica. Por lo anterior Meinardi afirma que un programa de formación docente integrada con la investigación didáctica demanda un conjunto de acciones compartidas por los docentes en formación, docentes en ejercicio, los investigadores en didáctica y formadores de formadores, esta articulación permite generar conocimiento teórico que puede orientar el desarrollo de nuevas experiencias y retroalimentar los procesos de formación docente.

Por otro lado Valbuena (2007) afirma que los modelos de formación inicial de profesores se pueden clasificar en cuatro tendencias; modelos tradicionales (transmisionistas), modelos tecnológicos, modelos espontaneistas, modelos de investigación y desarrollo profesional. Esta integración permite articular la investigación educativa con el diario vivir de los contextos educativos.

Para empezar, en los modelos tradicionales, el conocimiento del profesor se genera externamente a la realidad escolar, además procura que el profesor se limite a trasmitir la producción de científicos y especialistas quienes determinan qué contenidos debe aprender el profesor, teniendo prioridad el conocimiento disciplinar. En este sentido, Tardiff (2004, citado en Valbuena, 2007), afirma que en los programas de formación de profesores, predomina una importancia por la disciplina específica, presentando una desconexión entre los conocimientos teóricos y la acción profesional, en consecuencia las

\footnotetext{
${ }^{5}$ Es importante resaltar que dentro de la Licenciatura en biología de la Universidad Pedagógica Nacional se desarrolla investigación biológica, pedagógica y didáctica
} 
Bio - grafia. Escritos sobre la Biología y su Enseñanza. ISSN 2027

Edición Extraordinaria. p.p. $871-881$

Memorias del VIII Encuentro Nacional de Experiencias en Enseñanza de la Biología y la Educación Ambiental. III Congreso Nacional de Investigación en Enseñanza de la Biología.

prácticas de los docentes son insuficientes. Para Valbuena (2007) los profesores formados con este modelo no cuentan con un conocimiento profesional propio que lo diferencie de otros profesionales, puesto que creen, que el conocimiento disciplinar es suficiente para los procesos de enseñanza-aprendizaje.

Otro de los modelos de formación es el tecnológico, este modelo se basa en la implementación de fórmulas generales, técnicas y métodos producidos por grupos de especialistas. Para Porlán y Rivero (1998) el conocimiento profesional en este modelo, se entiende como un conocimiento técnico constituido por los conocimientos de las disciplinas específicas y el conocimiento de competencias y técnicas para la intervención en el aula.

Por otro lado el modelo espontaneista, necesita solamente habilidades personales apoyándose en la experiencia, en ese sentido si el individuo posee cualidades del "oficio de enseñar" está facultado para aprender a educar. Para Porlán y Rivero (2003), en este modelo, prima la acción sobre la reflexión, y la intervención sobre la planificación y el seguimiento, además se considera el aprendizaje profesional es como un proceso que ocurre espontáneamente en condiciones adecuadas.

Finalmente el modelo de investigación y desarrollo profesional, el cual se basa en el análisis de perspectiva práctica y la reflexión de esta, para la reconstrucción social y un enfoque alternativo, opta por la integración entre la teoría práctica, para reflexionar y encontrar soluciones a los problemas de la realidad social. Según Valbuena (2007) el modelo del profesor-investigador propone abordar la formación del profesorado desde el conocimiento profesional construido a partir de la reflexión de y para la práctica. En este modelo el conocimiento profesional se entiende como un proceso integración de diferentes conocimientos para la resolución de problemas de la práctica docente que permiten la toma de decisiones en diferentes contextos escolares.

\section{Formación investigativa de profesores}

Escobar (2012) menciona que el desarrollo de un profesor como investigador permite generar ambientes de aprendizaje donde la indagación y el cuestionamiento forman parte de la cotidianidad. En este proceso, el profesor y los estudiantes se preguntan, se cuestionan y proponen elementos que pueden generar cambios dentro de su mismo contexto. 
Bio - grafia. Escritos sobre la Biología y su Enseñanza. ISSN 2027

Edición Extraordinaria. p.p. $871-881$

Memorias del VIII Encuentro Nacional de Experiencias en Enseñanza de la Biología y la Educación Ambiental. III Congreso Nacional de Investigación en Enseñanza de la Biología.

Porlán y Rivero (2003), afirman que el profesor como un profesional investigativo, proviene de la especificidad de cada uno de los contextos a los que se acerca, puesto que cada uno de estos, tiene problemáticas específicas que corresponden a problemas relevantes y cuya investigación permite la organización, construcción y desarrollo del saber docente. Estos problemas tienen un carácter práctico, en este sentido, es que el profesor debe dar a conocer todo su conocimiento el cual le permitirá desarrollar, solucionar y profundizar en dichos problemas.

Debido a lo anterior, los diferentes contextos educativos, exigen que la formación de un profesor se conciba como un proceso de desarrollo profesional vinculado investigación educativa para la búsqueda de un cambio curricular. Según Escobar (2012), este proceso se basa en la integración de cuatro tipos de saberes: el primero es el saber experiencial del profesor, el segundo son las concepciones ideológicas de corte radical y alternativo, conectadas con las tradiciones anti-autoritaristas con la crítica al desarrollismo capitalista. El cuarto son los nuevos enfoques del saber disciplinar asociado a la educación (psicología cognitiva, psicología social, etnografía, modelos didácticos ecológicos, etc.) Finalmente el saber científico (epistemología relativista, teoría de las revoluciones científicas, teoría del caos, teoría de la complejidad, etc.)

En ese sentido Porlán y Rivero (2003) afirman que los profesionales de la educación deben tener la facultad de reconocer problemas complejos y singulares en el aula, los cuales no son divisibles mediante la educación tradicional. Lo que se pretende con una formación basada en la investigación es que el profesional promueva en sí mismo un proceso de reflexión continuo, sobre el cual pueda desarrollar funciones de las cuales emerja todo su conocimiento profesional el cual, le ayude a integrar saberes contextuales adquiridos en la experiencia y saberes formalizados apoyados en la teoría (Gimeno, 1983)..

\section{Formación en investigación en el Proyecto Curricular de Licenciatura en Biología (PCLB).}

El PCLB es un proceso formativo sistemático, en donde se propone desarrollar habilidades y competencias que permiten al licenciado en Biología, el logro de los objetivos propuestos, como por ejemplo el diseño y ejecución de proyectos de investigación en educación, pedagogía, biología y saberes afines. Con estos objetivos, el futuro maestro debe desarrollar destrezas de tipo investigativo que implican el dominio de conocimientos de un área del saber, de procedimientos científicos y el razonamiento complejo (Amórtegui y 
Bio - grafia. Escritos sobre la Biología y su Enseñanza. ISSN 2027

Edición Extraordinaria. p.p. $871-881$

Memorias del VIII Encuentro Nacional de Experiencias en Enseñanza de la Biología y la Educación Ambiental. III Congreso Nacional de Investigación en Enseñanza de la Biología.

Valbuena, 2009). Por otro lado, dentro de la misión del Departamento de Biología se plantea como principio, "la pertinencia y rigurosidad de la investigación del más alto nivel acerca de los problemas y necesidades nacionales en el ámbito de su competencia" (p. 17).

El PCLB está formado por dos ciclos, el ciclo de fundamentación y el ciclo de profundización. El ciclo de fundamentación está constituido por ejes curriculares de primero a sexto semestre, en donde se presentan espacios académicos comunes y se realizan prácticas educativas integrales, las cuales parten de pequeños proyectos investigativos, originados desde; las relaciones de los espacios académicos, los núcleos integradores de problema (NIP) y el interés del estudiante. Por su parte el ciclo de profundización tiene una duración de cuatro semestres, al iniciar este ciclo los estudiantes deben optar por un énfasis y una línea de investigación dependiendo de sus intereses, en la cual desarrollarán su trabajo de práctica y posteriormente el trabajo de grado.

El sexto semestre es denominado eje curricular interacción, en el cual la formación pedagógica y didáctica se configura como un campo de trabajo más complejo, puesto que se posibilita una reflexión y acción para el beneficio de la praxis educativa con apoyo en procesos investigativos. Para esto se propone la construcción de una propuesta investigativa en el eje curricular interacción, desde la reflexión de los intereses de toda la trayectoria de la formación como profesor, esta reflexión debe dar como resultado una propuesta para el inicio de la práctica pedagógica en diferentes campos educativos y enfocados a una línea de investigación del departamento de biología.

\section{METODOLOGÍA}

La presente propuesta de investigación se encuentra en el marco de la investigación cualitativa, puesto que, la principal característica es su interés por captar la realidad social a través de la percepción que tiene el sujeto en un contexto específico (Bonilla y Rodríguez, 1997). De acuerdo con Cerda (2005) esta investigación se caracteriza por utilizar múltiples fuentes y métodos para estudiar un solo problema, característica de la presente propuesta. Además de esto pertenece a la investigación educativa, dado que busca cuestiones y problemas relativos a la naturaleza, epistemología, metodología, fines y objetivos en el ámbito del conocimiento educativo. 
Bio - grafia. Escritos sobre la Biología y su Enseñanza. ISSN 2027

Edición Extraordinaria. p.p. $871-881$

Memorias del VIII Encuentro Nacional de Experiencias en Enseñanza de la Biología y la Educación Ambiental. III Congreso Nacional de Investigación en Enseñanza de la Biología.

En este sentido, esta propuesta pertenece al paradigma cualitativo interpretativo, el cual presenta ideas de corrientes humanísticas-interpretativas, que se ocupa de la comprensión e interpretación de la realidad educativa desde los significados de las personas implicadas en los contextos educativos. Según Cerda (2005) este paradigma se caracteriza por el énfasis que hace en la aplicación de técnicas de descripción, clasificación y explicación. Este paradigma en la investigación educativa, enfatiza en la comprensión e interpretación de la realidad educativa desde los significados de las personas implicadas en los contexto educativos y estudia sus creencia, intenciones, motivaciones y otras característica del proceso educativo no observables directamente ni susceptible de experimentación (Arnal, Rincón y Latorre, 1992)

Para el desarrollo de esta propuesta, la información provendrá de fuentes de información documentales y las declarativas, las cuales son:

- Las declaraciones de formadores y futuros profesores de sexto semestre de la Licenciatura de Biología, sobre la construcción del proyecto de integración, durante las tutorías dedicadas a esta actividad. Para adquirir esta información se utilizará la técnica de los grupos focales y el instrumento de la entrevista. Los grupos focales se caracterizan por ser una reunión con un grupo de individuos seleccionados por los investigadores, teniendo en cuenta el objeto de la investigación, con el fin de discutir desde la experiencia personal la temática o hecho social de interés (Korman, 1986).El objetivo de la investigación a través de grupos focales, es la recolección de información en profundidad sobre lo que las personas opinan y hacen, relacionado con el tema a investigar (Prieto y Cerda, 2002).

- Se realizarán entrevistas a los formadores y futuros profesores sobre las concepciones que poseen acerca de la investigación en el marco de la formación profesores. En ese sentido, la entrevista es una conversación entre dos personas; el sujeto y el investigador, con el fin de profundizar en el objeto a investigar (Cerda, 2005)

- Se analizarán los proyectos de semestre construidos por futuros profesores en el eje curricular interacción, a partir del análisis documental siendo este la revisión y análisis de documentos escritos para encontrar información importante para el objeto de la investigación(Valbuena, 2013) 
Bio - grafia. Escritos sobre la Biología y su Enseñanza. ISSN 2027

Edición Extraordinaria. p.p. $871-881$

Memorias del VIII Encuentro Nacional de Experiencias en Enseñanza de la Biología y la Educación Ambiental. III Congreso Nacional de Investigación en Enseñanza de la Biología.

La sistematización y el análisis de información se realizarán a través del método de análisis de contenido. Para Ander-Egg (1995) el análisis de contenido, como un método de recopilación de información que permite estudiar el contenido, clasificando a partir de categorías establecidas por el investigador, teniendo como objetivo identificar de manera sistemática las categorías dentro de la información obtenida. El análisis de contenido según Piñuel (2002), tiene dos objetos, primero la elaboración y procesamiento de datos relevantes sobre las condiciones misma que han producido los textos, y el segundo es lograr la emergencia de aquel sentido latente que procede de las prácticas sociales y cognitivas.

Como instrumento de recolección de información se utilizara la entrevista. Para Ander-Egg (1995), Cerda (2005) y Gil et al. (1999) la entrevista es una conversación entre por lo menos dos personas, en la cual uno es el investigador y el otro u otros son los investigados, estos dialogan con algún arreglo o pautas que dirigen la conversación en sentido del interés de los sujetos. Por otro lado Cerda (2005) afirma que la entrevista es el acto de hacer preguntas a alguien con el propósito de obtener algún tipo de información. De acuerdo con este autor se establece que es una de las formas de obtener información que no es evidente por medio de la observación. "Podemos penetrar en el mundo interior del ser humano y conocer sus sentimientos, sus ideas y conocimientos" (Cerda, 2005 pág. 259) Según Osorio (2002) la entrevista cualitativa permite resaltar contenidos verbales de la interacción a la investigación, es fundamental la connotación del habla, las emociones, los sentimiento expresados a través de los gestos "El cómo se dice es tan importante como lo que se dice así la entrevista no solo proporciona relatos si no brinda información acerca el contexto" (Osorio, 2002).

Este proyecto de investigación se desarrollará en cuatro fases:

- Diseño y validación de instrumentos para la recolección de información

- Construcción de categorías de análisis

- Recolección de información

- Sistematización de datos y análisis resultados.

\section{RESULTADOS ESPERADOS}


Bio - grafia. Escritos sobre la Biología y su Enseñanza. ISSN 2027

Edición Extraordinaria. p.p. $871-881$

Memorias del VIII Encuentro Nacional de Experiencias en Enseñanza de la Biología y la Educación Ambiental. III Congreso Nacional de Investigación en Enseñanza de la Biología.

Como principal resultado se espera la caracterización de la formación investigativa de los estudiantes de sexto semestre de la Licenciatura en Biología de la Universidad Pedagógica Nacional. Específicamente se espera describir la construcción del proyecto de integración de sexto semestre de la Licenciatura en Biología, a partir del seguimiento en la construcción de esta actividad de integración de un grupo focal de los estudiantes de sexto semestre. Está activad es muy importante puesto que cierra el ciclo de fundamentación y abre el ciclo de profundización con la propuesta de un proyecto de investigación que se debe desarrollar en la práctica pedagógica.

Por otro lado se espera el análisis de los proyectos de semestre propuestos por el grupo focal identificado, con el fin de establecer las tendencias e intereses investigativos que presentan los estudiantes de sexto semestre de la licenciatura en biología de la Universidad

Además se espera identificar las concepciones de los formadores y estudiantes de sexto semestre sobre la investigación en el marco de la formación de profesores

Todo este proceso investigativo se construye con el fin de aportar en el proyecto denominado "Formación en investigación en futuros profesores de biología" del grupo de investigación Conocimiento Profesional del Profesor de Ciencias. Además de esto, se espera fomentar una reflexión frente la formación investigativa de los futuros profesores de Biología y de ser necesario un mejoramiento en cuanto a la formación investigativa al Proyecto curricular de licenciatura en Biología.

\section{BIBLIOGRAFÍA}

AMÓRTEGUI, E. y VALBUENA, E. (2009). Algunas relaciones entre investigación, práctica pedagógica y formación inicial: relatos de una experiencia. Revista Bio-grafia 2(1), 124-136.

ANDER-EGG, (1995). Técnicas de investigación social. Argentina: Editorial Lumen

ANDREU, J. (2011). Las técnicas de análisis de contenido: una revisión documentada. Universidad de Granada 
Bio - grafia. Escritos sobre la Biología y su Enseñanza. ISSN 2027

Edición Extraordinaria. p.p. $871-881$

Memorias del VIII Encuentro Nacional de Experiencias en Enseñanza de la Biología y la Educación Ambiental. III Congreso Nacional de Investigación en Enseñanza de la Biología.

ARNAL, J.; RINCÓN, D. Y LATORRE, A. (1992) Investigación educativa. Fundamentos y metodología. Editorial Labor.

BONILLA, E. \& RODRIGUEZ, P. (1997). La investigación en Ciencias Sociales. Más allá del dilema de los métodos. Ediciones Unidades. Grupo Editorial Norma.

CERDA, Hugo. (2005). Los elementos de la investigación. Colombia: El Buho

ESCOBAR, G. (2012). Formación investigativa en futuras profesoras. El caso de dos estudiantes en el contexto de la práctica profesional inicial en un programa de licenciatura en biología. Tesis para optar al título de magíster en Educación. Bogotá: Universidad Pedagógica Nacional.

GIMENO, J. (1983). El profesor como investigador en el aula: un paradigma de formación de profesores. Educación y Sociedad, 2, 51-73.

KORMAN (1986). The Focus Group Sensign. Dept. Of Sociology, SUNY at Stony Brook. New York.

MEINARDI, E. (2010). Educar en ciencias. Acerca de la formación docente. Editorial Paidos. Argentina

PRIETO Y CERDA (2002) Paso a paso en el diseño de un estudio mediante grupos focales. Escuela Andaluza de Salud Pública. Granada. Aten Primaria 2002. 15 de abril. 29 (6): 366373

PIÑUEL, J. (2002). Epistemología, metodología y técnicas de análisis de contenido. Estudios de Sociolingüística 3(1), 1-42.

PORLÁN, R. (1987). El maestro como investigador en el aula. Investigar para conocer, conocer para enseñar. Investigación en la Escuela, 1, 63-69

PORLÁN, R. y RIVERO, A. (2003). La naturaleza y organización del conocimiento profesional "deseable" del profesorado. Publicación es resultado parcial del Proyecto PB970737 financiado por la CICYT.. Sevilla: Universidad de Sevilla, Departamento de Didáctica de las Ciencias. 
Bio - grafia. Escritos sobre la Biología y su Enseñanza. ISSN 2027

Edición Extraordinaria. p.p. $871-881$

Memorias del VIII Encuentro Nacional de Experiencias en Enseñanza de la Biología y la Educación Ambiental. III Congreso Nacional de Investigación en Enseñanza de la Biología.

VALBUENA, E, (2007) El conocimiento didáctico del contenido Biológico: estudio de las concepciones disciplinares y didácticas de futuros docentes de la Universidad Pedagógica Nacional (Colombia), Universidad Complutense de Madrid, tesis doctoral, Madrid

VALBUENA; E, (2009) Línea de investigación Conocimiento Profesional del Profesor de Ciencias. Vol 2, No 2 Revista Bio-grafia, Departamento De Biología. Universidad pedagógica nacional.

VALBUENA, E. (2012). Formación en investigación en futuros profesores de biología. Proyecto presentado a la convocatoria interna para grupos con proyectos de investigación en educación y pedagogía. Universidad Pedagógica Nacional

VALENCIA, N \& GARZÓN, D. (2012). Una experiencia investigativa en formación inicial de futuros licenciados en biología de la Universidad Pedagógica Nacional. Revista EDUCYT. Vol. Extraordinario. 